\title{
Ciencias de la Información: formación, retos y nueva propuesta desde Venezuela
}

\author{
Leomar José Montilla Peña \\ Guillermo Pérez Reyes \\ Universidad Politécnica Territorial Andrés Eloy Blanco - UPTAEB, Venezuela
}

\section{ANÁLISIS / ANALYSIS}

\begin{abstract}
Resumen
Se reflexiona sobre la formación de profesionales de la información en Venezuela y los posibles aportes que estos profesionales pueden brindar a la sociedad y su proyección ante ella. El contenido está dividido en tres partes: la primera aborda los aspectos relacionados con la formación de profesionales en Ciencias de la Información en Venezuela; la segunda proyecta la formación venezolana en Ciencias de la Información en el futuro y la tercera medita sobre las perspectivas para los profesionales en Ciencias de la Información.
\end{abstract}

\section{Palabras clave}

Ciencia de la información; Programa de formación; Profesionales de la información; Venezuela

\section{Information Sciences: training, challenges and new proposal from Venezuela}

\begin{abstract}
It reflects on the training of information professionals in Venezuela and the potential contributions that these professionals can provide to society and its projection to it. The content is divided into three parts: the first deals with issues related to professional training in Information Sciences in Venezuela, the second project the training Venezuelan Information Sciences in the future and the third reflects on the prospects for professionals in Information Science.
\end{abstract}

Keywords

Information science; Training programme; Information professionals; Venezuela

\section{Introducción}

Las organizaciones de información en Venezuela como en otros países del mundo presentan características muy variables y especiales de acuerdo a su tamaño, orientación y los tipos de servicios que estas brindan lo que la convierten en un verdadero desafío para su gestión de forma óptima, sumado a ello la escasez de recursos capitales o partidas presupuestarias y los rápidos avances tecnológicos que ocasionan de forma gradual la desinstitucionalización en la mayoría de ellas, pareciendo viable que decaigan de importancia y convirtiéndolas más aun en organizaciones complejas e inexorables, sin embargo, la viabilización del fortalecimiento deberá lograrse como un bien común, con la intención de reducir la brecha tecnológica para el manejo eficiente de información y el conocimiento dentro de las organizaciones e instituciones del país. 
Por tal motivo, surge el programa de estudio relacionado con la formación de profesionales en Ciencias de la Información con el propósito de que las universidades venezolanas den esta formación y a su vez acudan a las modificaciones necesarias en el diseño curricular para poder reflejar los cambios socioculturales, políticos y económicos que está viviendo la nación, todo ello enmarcado dentro de la Misión Sucre y la Misión Alma Mater.

Al mismo tiempo buscar el crecimiento de la imagen y el reconocimiento de los profesionales de la información tan importante y necesaria como las demás carreras que ofertan las casas de estudios de educación superior, con todas sus características y atributos propios, contando con un cuerpo docente magno en conocimientos como base indispensable para la acción práctica en pro de dar repuestas a la problemática puntualizada y dejar de ver a los profesionales de información como simples entes custodiales o cualquier otra denominación que intente menos cavar la importancia de tan meritoria profesión.

El objetivo de este ensayo es presentar algunos argumentos validos para una reflexión sana y verdadera sobre la educación y la formación profesional en Venezuela con respecto a la Ciencias de la Información, sin ánimo de comprometer a personas o instituciones alguna de formación superior, de igual forma plantea abordar las perspectivas de las oportunidades y porvenir de los profesionales en Ciencias de la Información en las organizaciones de información públicas y privadas en Venezuela, aprovechando las condiciones de transformación y cambios paradigmáticos que vive el país, América Latina y el mundo.

\section{Formación de profesionales en Ciencias de la Información en Venezuela.}

Conforme con lo desarrollado hasta ahora y para profundizar en los aspectos de educación profesional es muy significativo abordar los rasgos de la formación de los profesionales de las Ciencias de la Información, por su amplio carácter disciplinario, pero no sin antes considerar el vocablo profesión la cual proviene del latín, "Professione" que significa acción y efecto de profesar, un oficio o empleo, fundamentándose en la educación, formación y perfeccionamiento con dedicación exclusiva e interesante del individuo a determinada actividad bajo diversas modalidades, aspectos o saberes del carácter científico y técnico precisos para realizar un conjunto de tareas, buscando además una comprensión más completa del ambiente de trabajo y de otros aspectos que hacen a la vida no sólo laboral sino también de cuestiones relativas a su desarrollo personal, social y cultural.

La génesis de la profesión viene dado desde que el hombre se organizó en comunas para lograr su sobrevivencia, desarrollando técnicas para las distintas actividades que fueron surgiendo, enfocándonos en las ideas de Hodges (1978) que exponía en su libro La Estratificación Social Las Clases en América, las estructuras de la sociedad resultan de especializaciones de una masa relativamente homogénea, pasando a ser heterogénea con la vinculación de los factores sociales, culturales, políticos, económicos, financieros, religiosos y los aspectos étnico que ha tenido que ver con el desenvolvimiento de la sociedad, lo que ha originado la multiplicidad de profesiones las cuales se van separando una de otras por la realización de subdivisiones.

La formación profesional puede precisarse pródigamente como la transmisión del conocimiento, habilidades y destrezas las cuales se proporcionan a través de la educación, la enseñanza, la formación y el aprendizaje, estando esta articulada e integrada, transformándose en un factor de suma importancia orientada a integrar y potenciar competencias aplicables a una variedad de situaciones laborales y áreas de ocupación.

Coexistiendo la formación en un proceso permanente a lo largo de la vida en conjunción con otras formas de educación, exigiendo una ineludible actualización para relacionarse con el entorno, la información y los medios de comunicación.

Freire (2006) en su capítulo No hay docencia sin discencia del libro de Pedagogía de la autonomía: Saberes necesarios para la práctica educativa, nos describe "que quien se está formando, desde el principio mismo de su experiencia formadora, se convenza de que enseñar no es trasmitir conocimiento, sino de crear las posibilidades de su producción o de su construcción" (p. 24).

En este orden de ideas, según la Declaración Mundial sobre la Educación Superior en el Siglo XXI, en el artículo 9, expresa que "las instituciones de educación superior deben formar a los estudiantes para que se conviertan en ciudadanos bien informados y profundamente motivados, provistos de un sentido crítico y capaces de analizar los 
problemas de la sociedad, buscar soluciones para los que se planteen a la sociedad, aplicar éstas y asumir responsabilidades sociales".

Partiendo de este artículo que enfatiza la función de las instituciones de educación superior se puede precisar implícitamente las fortalezas que debe poseer el Docente, entre ellas están la de ser un formador de la creatividad y del pensamiento crítico en la que debe generar en el estudiante las condiciones favorables para elaborar esquemas de pensamientos que le permitan interpretar las realidades de acuerdo a las experiencias previas.

Asimismo debe ser un motivador capaz de estimular a los estudiantes de crecer personal e intelectualmente, por otra parte, debe ser un socializador que lo involucre con la sociedad a la que en un futuro muy cercano deberá dar soluciones de acuerdo a los conocimientos adquiridos garantizando el desarrollo sostenible de la sociedad en general.

Por ello, la formación tiene que ir más allá de una orientación académica-tecnológica, también debe aplicar la práctica y el enfoque social centrándola en un nuevo modelo de formación, donde busque fomentar la experiencia personal y grupal, promoviéndola en un proceso reflexivo que lleve a las implicaciones éticas y políticas de actuación, articulando de esa forma el desarrollo por competencias orientadas a la creciente necesidades informacionales de los usuarios con una alta capacidad crítica y colindante con los servicios personalizados.

Cabe enfatizar que los efectos causados por la revolución industrial, la revolución científica, los acontecimientos bélicos que ha experimentado la humanidad y el naciente postmodernismo de la década de los 70 ha producido un gran flujo de información, residiendo ésta en la competencia laboral en su máxima expresión para dar repuesta eficaz al mercado laboral, aceptando el desafío de los nuevos paradigmas que se encuentran en ellos, por tal motivo la Ciencias de la Información surge como una profesión postmoderna que se ocupa de las organizaciones de información o instituciones públicas y privadas para el manejo y dominio de la información registrada y su transmisión por medio de las tecnologías para la búsqueda y recuperación de la información a través de la comunicación, contribuyendo a la satisfacciones de las necesidades informacionales del o los individuos.

Motivado además por lo que sucintamente delinea Castells (2005) "En el nuevo modo de desarrollo informacional, la fuente de la productividad estriba en la tecnología de la generación del conocimiento, el procesamiento de la información y la comunicación de símbolos" (p. 43). Dejando claro que la información es el principal recurso para la adquisición y aplicación del conocimiento y la toma de decisiones. Por tal motivo, el profesional de la información debe guardar relación con la organización donde se encuentre porque ello permitirá ser efectivo y eficiente en concordancia con la misión, visión y objetivos de ella, que es lo que nos concierne en esta ocasión.

La existencia de la información confiable y el acceso de la sociedad a ella, es, quizás, el recurso más poderoso para modificar el curso de muchos procesos socio productivos del país. La concepción y puesta en práctica de un nuevo modelo de justicia social el cual tiene como condición necesaria el reconocimiento de los problemas y sus alcances económicos sociales, porque ninguna política sensata y justa puede fundarse prudencialmente, sin el puntal inexcusable que debe representar los sistemas de información en todo el contexto científico, académico y social.

Por ello, surge el Programa de Formación de Ciencias de la Información con el ánimo de formar profesionales de información en toda la extensión de la palabra, con la pretensión de suministrar a esos profesionales los instrumentos necesarios para el manejo eficiente de la información registrada, ya que hemos pasado de una economía generadora de productos a una orientada a los servicios informacionales.

El Diseño Curricular de Ciencias de la Información desarrollado hasta ahora en Venezuela se acopla al mecanismo de acreditación de títulos de grado del MERCOSUR, donde varias universidades de los países que lo integran han participado en la constitución de programas de formación universitaria que atiendan y concilie en los intereses comunes de este espacio de integración multinacional, estribando además el perfil de competencias para los profesionales de la información. De igual forma se considera las competencias desarrolladas en el EUROREFERENCIAL que es donde se vislumbra el desarrollo de las Euro competencias en Información y Documentación de acuerdo al contexto de la Unión Europea y de esta forma evitar intentos aislados.

Enmarcado por demás en una formación didáctica, flexible, integral, participativa, crítica e innovadora con una base solida que permite realizar cambios en su estructura dinámica, con un enfoque interdisciplinario, multidisciplinario y transdisciplinario (aunque este último tiende alarmar a muchos cientificistas) articuladas con el ser, conocer, hacer y 
el convivir en un proceso multidimensional de valoración donde el objetivo es fomentar la acción trasformadora y la corresponsabilidad de todos los actores.

El Programa Nacional de Formación en Ciencias de la Información está enmarcado en un área de conocimiento que reúne a su vez cuatro disciplinas científicas formalmente constituidas (Bibliotecología, Archivología, Museología y Ciencia de la Información), este hecho resulta innovador en nuestro sistema de educación superior por tratarse de un espacio interdisciplinario que declara, implícitamente o explícitamente, la información como objeto de estudio y que ese objeto de estudio sirve para satisfacer las necesidades informacionales -contenidos- que demandan las personas, es decir, son disciplinas científicas orientadas a analizar y resolver problemas que atañen directamente a la dinámica social; actualmente la comunidad científica internacional considera estas disciplinas parte de las ciencias sociales y reconoce además el valor epistemológico de cada disciplina por separado.

El Programa Nacional de Formación en Ciencias de la Información tiene como finalidad formar profesionales comprometidos con el desarrollo social del país, que respondan a una nueva ciudadanía democrática, gerentes innovadores, investigadores con vocación de servicio, capaces de dirigir organizaciones de información utilizando principios y herramientas de gestión de calidad para generar y administrar servicios y productos de información; coordinar y aplicar procesos documentales utilizar y gestionar herramientas tecnológicas para satisfacer las necesidades informacionales de las personas y la comunidad.

Estos profesionales son capaces de utilizar métodos de investigación orientados a conocer la realidad sociocultural y organizacional que le permitan responder a los requerimientos informacionales de las comunidades de usuarios, con competencia para elaborar planes de orientación, formación y promoción para el desarrollo de la cultura informacional y para afianzar los valores y la identidad nacional en los ámbitos en que se desenvuelve.

Al determinar la exigencia en un nuevo proceso de formación de profesionales de la información no se está desconociendo la contribución de las universidades tradicionales venezolanas donde se da la formación en bibliotecología, archivología y Museología, como tampoco los profesionales que de ella egresan y algunos resultados obtenidos dentro del campo científico de la información en el país, pero, se hace imperioso el mejorar los artefactos para el manejo de la información registrada, ello motivado por las estructuras sociales, las necesidades, problemáticas, exigencias y potencialidades de las organizaciones de información así como las de instituciones públicas o privadas de acuerdo a su contexto.

El cambio en los estudios de pregrado y postgrado tienen que ser cuidadosamente orientados hacia las necesidades de la sociedad y ser evaluado por los responsables del diseño curricular, las autoridades ministeriales y universitarias, docentes y estudiantes para la acreditación de un programa de formación de calidad donde el mejoramiento continuo vaya desde la reasignación de los recursos en apoyo a los diseños curriculares hasta la organización docente y su cambio de rol para la formación de sus estudiantes.

\section{La formación venezolana en Ciencias de la Información en el futuro.}

El enfoque tiene que transformase en un acatamiento a corto y mediano lapso para alcanzar la formación óptima, que origine la investigación, la transformación de las organizaciones de información, la innovación en tecnologías para la comunicación, el bienestar y satisfacción informacional de los usuarios mediante la modernización de los diseños y ofertas de servicios, así como la promoción de un profesional competitivo que sea aceptado ante la sociedad, científicamente y tecnológicamente preparado, organizacionalmente administrado y socialmente equitativo para evitar comentarios anímicos antes el imaginario social. Recordando nuevamente a Honges (1978). .... la hora de determinar "qué es lo que él es" (p.32).

Con una determinación de renovación en los procesos informacionales que respondan positivamente a las necesidades informacionales de las comunidades de usuarios, ponderando temas dirigidos a mejorar la construcción o producción del conocimiento científico de la información y las capacidades técnicas, el liderazgo, la gestión de organizaciones de información, suministrando el entendimiento sistémico de la importancia social.

Un diseño curricular proveído en el perfeccionamiento para formar profesionales de información en un futuro de coyuntura y bienestar, enfrentándose a retos sin evadir la responsabilidad de la vida y en el combate del 
desconocimiento informacional, engrosando los diseños de los servicios en las organizaciones de información para evitar la deserción y desuso de los usuarios permitiendo aumentar el acceso a la información en las distintas condiciones de los servicios y procesos operacionales a través del impacto que este tenga ante la sociedad.

Por otro lado, el profesional de la información debe realizar sus investigaciones y su práctica social dentro de su municipio y/o las organizaciones de información a través de proyectos organizacionales y comunitarios orientados a mejorar continuamente el acceso a la información a las comunidades de usuarios, dando respuestas reales a las problemáticas sociales que vive el país basada en la equidad y justicia social logrando así la consolidación del nuevo proyecto de país y la integración latinoamericana.

\section{Perspectivas para los profesionales en Ciencias de la Información.}

Los profesionales de la información ante el posicionamiento en la sociedad tendrá múltiples ofertas de empleo, sin embargo este estará antes que nada estrechamente ligado a las instituciones y sociedades donde se involucre para profundizar en la comprensión de las necesidades informacionales requeridas por las sociedades discursivas según sea el caso, en una práctica social normalizada institucionalmente, que incluye tanto aquellas actividades, gerenciales, técnicas y operativas del profesional de información que son susceptibles de ser reenumeradas, inspeccionadas y evaluadas por la institución y la sociedad a la que prestará su servicio.

Ciertamente el profesional de la información debe poseer un buen conocimiento de lo que es la gestión de organizaciones de información, que este conocimiento provenga de una educación formal de la ciencia, pero también se hace imperioso de que los especialistas de la información se empoderen del lenguaje natural de las ciencias o disciplinas donde prestarán sus servicios profesionales como un requisito mínimo indispensable, no para dotarse otra profesión, sino para apoderarse del discurso de estos.

Además de familiarizarse con las principales fuentes de información tradicionales como digitales en diferentes idiomas, los servicios de información, las principales bases de datos y otras organizaciones de información que guarden relación al enfoque de la organización donde se encuentre, debido a la inter, multi y transdisciplinariedad y los ámbitos geográficos de las ciencias hoy en día, de esta manera se mantendrá a tono con los diferentes recursos que existan, teniendo en claro que no es una labor intrascendente, sino, transcendente en todos sus aspectos profesional para la resolución de problemas y sobre todo para la toma de decisiones.

Las organizaciones de información son espacios dinámicos de la comunicación, lo que exige una actividad intelectual constante e innovadora con amplia participación de responsabilidad individual y colectiva en beneficio de los usuarios donde el profesional de la información debe poner en práctica de forma racional el conocimiento, habilidades, técnicas, ética y la retroalimentación de forma directa e indirecta, así como los instrumentos desarrollados e inéditos en la Ciencias de la Información para logra la satisfacción de las necesidades informacionales de los usuarios.

Corresponden en formular mejores estrategias gerenciales, políticas, planes, proyectos $u$ ofertas de servicios que permitan dominar la información, adecuándolos a los objetivos y desempeños de las organizaciones de información conexo con las circunstancia y más allá de las satisfacciones de las necesidades informacionales de sus usuarios a través de potenciales técnicas o métodos que permitan alcanzar los resultados y a la misma vez perfeccionar la Ciencias de la Información.

Bajo un nuevo modelo holístico y de pensamiento complejo, basado en el uso intensivo de los recursos y servicios disponibles en la organizaciones de información y tras los muros, con una visión ecológica de la información, debido a que estamos viviendo la era de la información, la cual "Se caracteriza cada vez más por los senderos electrónicos digitales que se irradian en todas direcciones. El acceso instantáneo a la información está al alcance de todos lo que los necesite. La reducción de la barreras tiempo-distancia a la velocidad de la luz es un hecho" Timmerman (2000, p. 34).

Partiendo de la premisa descripta, los profesionales de la información deben priorizar las acciones tendientes a la gestión de tecnologías, administración y organización de la información de acuerdo al sujeto real del proceso: el usuario de la información y la sociedad a la que pertenece, ya que la información es un concepto abstracto que se 
puede implementar de muchas maneras diferentes. Capurro (2008) señala que "la noción de la información se refiere en muchas de las significaciones científicas analizadas a resortes" (p.18). Considerando por demás que la información es la elemento primordial para el éxito de toda institución y de las actividades de los individuos, transferibles a un conocimiento.

Un aspecto importante que está marcando tendencia es el "Análisis del dominio" (Hjørland y Albrechtsen, 1995), cuyo fundamentos radica en estudiar los dominios de conocimientos como comunidades hablantes o del pensamiento, esto con el propósito de desarrollar servicios y productos de información dirigidos a comunidades discursivas con necesidades de información puntuales y estilos cognitivos particulares. Es por ello que las temáticas: organización y representación de la información, la gestión de información y del conocimiento, los estudios métricos de la información, la búsqueda y recuperación de la información, los estudios de necesidades y uso de la información en las diferentes organizaciones de información, entre otros, deben desarrollarse con esta nueva mirada, el "Análisis del Dominio".

Otro aspecto primordial que debe poseer el profesional de la información en su rol técnico operativo, es el compromiso que adquiere con la organización, pero sobre todo que sean capaces de generar cambios efectivos en las organizaciones sus unidades y servicios de información, independientemente de la función que le toque desempeñar en el futuro. Profesionales capaces de gestionar, organizar y manejar información registrada para sus usuarios con el propósito de ejecutar acciones efectivas en cualquiera de los campos del quehacer profesional.

\section{A modo de conclusión}

A la luz de los análisis efectuados hay que matizar lo que se dijo al principio sobre la necesidad de formación de profesionales de la información, percibiendo en la sociedad su importancia, una reflexión sobre los resultados que se obtendrán con la certificación de profesionales de la información permite entresacar algunas ideas que ponen de manifiesto que estamos ante un Diseño Curricular de vanguardia y que tiene que ser suficientemente aprovechado por todos sus actores en todas sus potencialidades.

Como reflexión final y en relación con lo anteriormente apuntado sobre la formación y la necesidad de transformación de los profesionales de la información, sería interesante que en las próximas evaluaciones del Diseño Curricular de Ciencias de la Información que se desarrolla en Venezuela contemplara en él todas las ramas de la actividad informacional para optimizar el Programa de Formación Nacional de Ciencias de la Información.

\section{Bibliografía}

Capurro, R. (2008). Pasado, presente y futuro de la noción de información. Extraído 06 de Diciembre de 2011 desde http://www.capurro.de/leon.pdf.

Castell M. (Ed.). (2005). La Era de la Información (Vol. 1 La sociedad Red). Mexico: Siglo XXI.

Freire, P. (2006). Pedagogía de la autonomía: Saberes necesarios para la práctica educativa. México: siglo XXI.

Hjørland, B. y Albrechtsen, H. (1995). Toward a new horizon in Information and Science Domain Analysis. Journal of the American Society for Information Science, 46(6), 400-425. Extraído el 07 de Diciembre de 2011 desde

http://www.mendeley.com/research/toward-new-horizon-information-science-domain-analysis-1/

Honges, H. (1974). La estratificación social: Las clases en América. Madrid: Tecnos.

Timmerman, F. (2000). Como ganar el desafío de la información. España: Deusto S.A.

UNESCO. (1993). Declaración Mundial sobre la Educación Superior en el Siglo XXI: Visión y Acción. [Página web en línea] Extraído el 06 de Enero de 2012 desde http://www.unesco.org/education/wche/declaration.shtml

\section{Datos de los autores}




\section{Leomar José Montilla Peña}

Licenciado en Bibliotecología y Ciencia de la Información. Egresado de la Universidad de la Habana, Faculta de Comunicación, La Habana, Cuba. Encargado de la Biblioteca Especializada del Instituto Nacional de Investigaciones Agrícola, Unidad Ejecutora Lara, Venezuela. Profesor Instructor del Programa de Formación en Ciencias de la Información de la Universidad Politécnica Territorial Andrés Eloy Blanco, Barquisimeto, Venezuela. Integrante del Comité Interinstitucional para el Fortalecimiento de la Gestión del Programa Nacional de Formación en Ciencias de la Información, Ministerio del Poder Popular para la Educación Universitaria, Caracas, Venezuela.

leomonp@gmail.com

\section{Guillermo Pérez Reyes}

Licenciado en Bibliotecología y Ciencia de la Información. Egresado de la Universidad de la Habana, Faculta de Comunicación, La Habana, Cuba. Coordinador de Procesos Técnicos de la Biblioteca de la Universidad Politécnica Territorial Andrés Eloy Blanco, Barquisimeto, Venezuela. Profesor Instructor del Programa de Formación en Ciencias de la Información de la Universidad Politécnica Territorial Andrés Eloy Blanco, Barquisimeto, Venezuela. Integrante del Comité Interinstitucional para el Fortalecimiento de la Gestión del Programa Nacional de Formación en Ciencias de la Información, Ministerio del Poder Popular para la Educación Universitaria, Caracas, Venezuela.

pguillermoandres@gmail.com

Recibido - Received: 2012-02-28

Aceptado - Accepted: 2012-03-30

\section{(cc) BY-NC-ND}

This work is licensed under a Creative Commons

Attribution-Noncommercial-No Derivative Works 3.0 United States License.

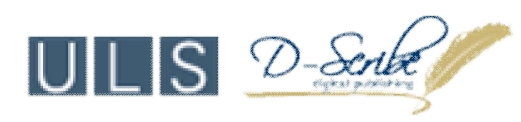

This journal is published by the University Library System of the University of Pittsburgh as part of its $\underline{D-S c r i b e}$ Digital Publishing Program and is cosponsored by the University of Pittsburgh Press. 